\title{
The complete genome sequence of Hafnia alvei A23BA; a potential antibiotic-producing rhizobacterium
}

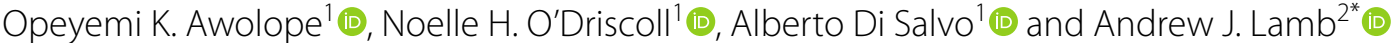

\begin{abstract}
Objectives: The urgent need for novel antibiotics cannot be overemphasized. Hafnia alvei A23BA was isolated from plant rhizosphere as part of an effort to recover novel antibiotic-producing bacterial strains from soil samples. The genome of the isolate was sequenced to facilitate mining for potential antibiotic-encoding biosynthetic gene clusters and to gain insights into how these gene clusters could be activated.

Data description: Here, we report the complete genome sequence of $H$. alvei A23BA obtained from the hybrid assembly of Illumina HiSeq and GridION reads. The genome, consisting of a circular chromosome and a circular plasmid, is $4.77 \mathrm{Mb}$ in size with a GC content of $48.77 \%$. The assembly is $99.5 \%$ complete with genomic features including 4,217 CDSs, 125 RNAs, and 30 pseudogenes. Thiopeptide, beta-lactone, siderophore, and homoserine lactone biosynthetic gene clusters were also identified. Other gene clusters of interest include those associated with bioremediation, biocontrol, and plant growth promotion- all of which are reported for $\mathrm{H}$. alvei for the first time. This dataset serves to expedite the exploration of the biosynthetic and metabolic potentials of the species. Furthermore, being the first published genome sequence of a soil isolate, this dataset enriches the comparative genomics study of $H$. alvei strains.
\end{abstract}

Keywords: Hafnia alvei, H. alvei, Genome mining, Biosynthetic gene clusters, Antibiotics, Rhizobacteria, Bioremediation, Biocontrol, Plant growth-promoting rhizobacteria

\section{Objective}

Bacterial secondary metabolites are invaluable sources of novel bioactive compounds. Many clinically useful antibiotics were derived from the secondary metabolites of soil dwelling bacteria [1]. However, only a small fraction of all known species have had their metabolites exploited in this way [2]. To this end, we sought to isolate novel antibiotic-producing bacterial strains from soil samples collected from the rhizosphere as antibiosis occurs naturally within it [3]. H. alvei A23BA was recovered as part of this effort.

\footnotetext{
${ }^{*}$ Correspondence: a.lamb@rgu.ac.uk

${ }^{2}$ Graduate School, Robert Gordon University, The Ishbel Gordon Building, Garthdee Road, Aberdeen AB10 7QE, Scotland
}

Full list of author information is available at the end of the article
Hafnia alvei is a Gram-negative, rod- shaped, facultatively anaerobic psychrotrophic bacterium. It is commonly isolated from clinical materials, gastrointestinal tract of animals, plant surfaces, soil, and water [4]. Some strains are commensals of the gastrointestinal tract while others are opportunistic pathogens implicated in both nosocomial and community-acquired infections $[5,6]$. It is almost never associated with antibiotic production except for the antimicrobial activities reported for a strain isolated from the gut of honeybees [7]. Phylogenetic studies of this little-known species have shown its pan-genome to be open and dynamic with each strain possessing sets of unique genes [8]. Unique gene acquisition is mainly by horizontal gene transfer, and it reflects the adaptation of strains to their remarkably diverse natural habitats. Strains show considerable metabolic

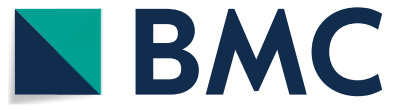

(c) The Author(s) 2021. This article is licensed under a Creative Commons Attribution 4.0 International License, which permits use, sharing, adaptation, distribution and reproduction in any medium or format, as long as you give appropriate credit to the original author(s) and the source, provide a link to the Creative Commons licence, and indicate if changes were made. The images or other third party material in this article are included in the article's Creative Commons licence, unless indicated otherwise in a credit line to the material. If material is not included in the article's Creative Commons licence and your intended use is not permitted by statutory regulation or exceeds the permitted use, you will need to obtain permission directly from the copyright holder. To view a copy of this licence, visit http://creativeco mmons.org/licenses/by/4.0/. The Creative Commons Public Domain Dedication waiver (http://creativecommons.org/publicdomain/ zero/1.0/) applies to the data made available in this article, unless otherwise stated in a credit line to the data. 
pathway diversity and varied biosynthetic potentials because of the open pan-genome making them good mining candidates for novel metabolites.

Consequently, the genome of $H$. alvei A23BA was sequenced to enable mining for potential antibioticencoding secondary metabolite biosynthetic gene clusters (smBGCs) that show little or no homology to known smBGCs. Furthermore, assembled genomes of $H$. alvei in public repositories are typically of clinical, human or food isolates, to the best of our knowledge, the complete genome sequence of $H$. alvei A23BA represents the first published complete genome sequence of a soil isolate.

\section{Data description}

H. alvei A23BA was recovered from the rhizosphere of a garden plant in Aberdeen, Scotland (57.101 N 2.078 W). It was isolated using an ultra-minimal substrate medium (Data file 1) [9]. Upon isolation and strain purification, isolate was cultivated in nutrient broth (Oxoid, UK) at $37{ }^{\circ} \mathrm{C}$ for $24 \mathrm{~h}$. Overnight culture was centrifuged and gDNA was extracted from pellets with the DNeasy ${ }^{\circledR}$ Ultraclean ${ }^{\circledR}$ Microbial Kit for DNA Isolation (Qiagen, UK). Isolate was preliminarily identified by $16 \mathrm{~S}$ rRNA gene sequence comparison as $H$. alvei with $99 \%$ identity score.

Libraries were subsequently prepared from extracted gDNA by MicrobesNG (Birmingham, UK) for whole genome sequencing. For Illumina sequencing, libraries were prepared using the Nextera XT Library Prep Kit (Illumina, USA) and sequenced with the Illumina HiSeq system using a 250 bp paired end protocol. For GridION (Oxford nanopore) sequencing, libraries were prepared with Oxford nanopore SQK-RBK004 kit and/or SQKLSK109 kit with Native Barcoding EXP-NBD104/114 (ONT, UK) using 400-500 ng HMW DNA. Sequencing was performed on a FLO-MIN106 (R.9.4 or R.9.4.1) flow cell in a GridION (ONT, UK).

Illumina sequencing run produced 4,973,530 short reads that were trimmed and paired using Trimmomatic [10] v0.30 with a sliding window quality cut-off of Q15. Ninety eight percent of reads were retained, and quality was assessed with FastQC [11] v0.11.8. Mean phred score across each base position was assessed with MultiQC [12] and found to be $\geq 28$ (Data file 2) [13]. GridION sequencing run produced 18,642 reads with the mean read quality score of 10.5 (data file 3) [14] as assessed with NanoStat [15]. Paired short reads and long reads from GridION sequencing were assembled with Unicycler [16] v0.4.8.0. Assembly quality was assessed with QUAST [17] v5.0.2- two contigs (one chromosome and one plasmid) were identified with a total length of $4,772,047 \mathrm{bp}, \mathrm{N} 50$ value of 4,687,005 bp and \#N's per $100 \mathrm{kbp}$ value of 0 (data file 4) [18]. Assembly completeness was assessed with BUSCO [19] v3.0.2 and found to be $99.5 \%$ (data file 5) [20]. Identity was confirmed as $H$. alvei by ANI analysis using the FastANI tool [21], with the ANI value of 97.8167. Gene and functional annotations were performed with PGAP [22] v4.11 and RASTtk [23]; pathways analyses were performed using the KEGG database [24] Rel 93.0 and the eggNOG mapper [25] vs 2.0.0. smBGCs were identified with antiSMASH [26] v5.0. Genome map was drawn with CGView [27] and presented in data file 6 [28].

In summary, the complete genome sequence of $H$. alvei A23BA is $4,772,047$ bp in size with the overall GC content of $48.77 \%$ and sequencing coverage of $256.0 \mathrm{x}$. It comprises of one circular chromosome $(4,687,005 \mathrm{bp}$; GC content $48.8 \%$ ) and one circular plasmid (85,042 bp; GC content 47.2\%). Genomic features include 4,217 CDSs, 25 rRNA, 92 tRNA, 8 ncRNA, 30 pseudogenes and 2 CRISPRs. Thiopeptide, beta-lactone (both showing little or no homology to known smBGCs) and siderophore smBGCs were identified (data file 7) [29]. Thiopeptides and beta-lactones are known for their antibiotic and/or anticancer activities [30,31], while siderophores are used clinically as "Trojan horse" to deliver antibiotics to antibiotic resistant bacteria [32]. Gene clusters commonly associated with bioremediation, biocontrol, environmental adaptation, and plant growth promotion were also identified (data file 8) [33]. Please see Table 1 for links to data files 1-8.

Given the quality control measures applied and results of analyses undertaken, we believe Hafnia alvei strain A23BA chromosome, complete genome [34] represents a high-quality dataset that would expedite the exploration of the biosynthetic and metabolic potentials of $H$. alvei A23BA and would also enrich the comparative genomics study of $H$. alvei strains.

\section{Limitations}

This dataset was generated from a hybrid assembly to ensure accuracy and completeness. Furthermore, the hybrid assembler (Unicycler) autocorrects read errors and polishes final assemblies several times to ensure accuracy. Annotations and metabolic pathway analyses were carried out with robust and validated bioinformatics tools, and smBGCs were identified with the most comprehensive genome mining tool to date. Therefore, the authors are currently unaware of any limitations of the data. 
Table 1 Overview of data files/data sets

\begin{tabular}{|c|c|c|c|}
\hline Label & Name of data file/data set & File types (file extension) & $\begin{array}{l}\text { Data repository and identifier (DOI } \\
\text { or accession number) }\end{array}$ \\
\hline Data file 1 & $\begin{array}{l}\text { Composition of ultra-minimal substrate } \\
\text { growth medium }\end{array}$ & Portable Document Format file (.pdf) & $\begin{array}{l}\text { https://doi.org/10.6084/m9.figshare.12781 } \\
\text { 193.v [9] }\end{array}$ \\
\hline Data file 2 & Quality distribution of Illumina reads & Joint Photographic Experts Group file (.jpg) & $\begin{array}{l}\text { https://doi.org/10.6084/m9.figshare.12643 } \\
\text { 961.v1 [13] }\end{array}$ \\
\hline Data file 3 & $\begin{array}{l}\text { Basic quality statistics of GridION sequenc- } \\
\text { ing data }\end{array}$ & Portable Document Format file (.pdf) & $\begin{array}{l}\text { https://doi.org/10.6084/m9.figshare.12781 } \\
\text { 280.v1 [14] }\end{array}$ \\
\hline Data file 4 & Quast report & Portable Document Format file (.pdf) & $\begin{array}{l}\text { https://doi.org/10.6084/m9.figshare.12781 } \\
\text { 289.v1 [18] }\end{array}$ \\
\hline Data file 5 & Short BUSCO summary & Joint Photographic Experts Group file (.jpg) & $\begin{array}{l}\text { https://doi.org/10.6084/m9.figshare.12643 } \\
\text { 937.v1 [20] }\end{array}$ \\
\hline Data file 6 & $\begin{array}{l}\text { Circular representation of H. alvei A23BA } \\
\text { genome }\end{array}$ & Joint Photographic Experts Group file (.jpg) & $\begin{array}{l}\text { https://doi.org/10.6084/m9.figshare.12643 } \\
\text { 949.v1 [28] }\end{array}$ \\
\hline Data file 7 & $\begin{array}{l}\text { Predicted smBGCs of interest in H. alvei } \\
\text { A23BA genome }\end{array}$ & Portable Document Format file (.pdf) & $\begin{array}{l}\text { https://doi.org/10.6084/m9.figshare.12781 } \\
\text { 274.v1 [29] }\end{array}$ \\
\hline Data file 8 & $\begin{array}{l}\text { Other gene clusters of interest in } \mathrm{H} \text {. alvei } \\
\text { A23BA genome }\end{array}$ & Portable Document Format file (.pdf) & $\begin{array}{l}\text { https://doi.org/10.6084/m9.figshare.13309 } \\
\text { 049.v1 [33] }\end{array}$ \\
\hline Data set 1 & Illumina and GridION sequencing reads & Fastq file (.fastq.gz) & $\begin{array}{l}\text { https://identifiers.org/ncbi/insdc.sra:SRP25 } \\
1948 \\
{[35]}\end{array}$ \\
\hline Data set 2 & Genome assembly of $H$. alvei A23BA & Fasta file (.fna) & $\begin{array}{l}\text { https://identifiers.org/ncbi/insdc } \\
\text {.gca:GCF_011617105.1 [36] }\end{array}$ \\
\hline
\end{tabular}

\section{Abbreviations}

GC: Guanine-Cytosine; CDSs: Coding sequences; RNA: Ribonucleic acid; rRNA: Ribosomal ribonucleic acid; tRNA: Transfer ribonucleic acid; ncRNA: Non-coding ribonucleic acid; CRISPRs: Clustered regularly interspaced short palindromic repeats; DNA: Deoxyribonucleic acid; gDNA: Genomic deoxyribonucleic acid; smBGCs: Secondary metabolite biosynthetic gene clusters; ONT: Oxford Nanopore technology; HMW: High molecular weight; \#N's: Average number of uncalled bases; ANI: Average nucleotide identity.

\section{Acknowledgements}

Genome sequencing was provided by MicrobesNG (http://www.microbesng uk) which is supported by the BBSRC (Grant Number BB/L024209/1).

\section{Authors' contributions}

The project was conceived and designed by OKA and AJL. Data acquisition was performed by OKA. Data analysis and interpretation was performed by OKA, NHO, ADS and AJL. The project was jointly supervised by NHO, ADS and AJL. AJL was the principal investigator. The manuscript was written by OKA and revised by $\mathrm{NHO}, \mathrm{ADS}$ and AJL. All authors read and approved the final manuscript.

\section{Funding}

This project was supported by Tenovus Scotland (Grant Number G16.04)

\section{Availability of data and materials}

Data files $1-8$ described in this Data note can be freely and openly accessed on Figshare (https://figshare.com/) [9, 13, 14, 18, 20, 28, 29, 33]. Data sets 1 and 2 can be freely and openly accessed on the NCBI database. Illumina and GridION reads generated have been deposited in the Sequence Read Archive under accession number SRP251948 (Data set 1) [35]. The genome assembly of $H$ alvei A23BA has been deposited in GenBank under accession number GCF_011617105.1 (Dataset 2) [36]. The BioProject accession number for the entire project is PRJNA610978. See Table 1 and references for details and links to the data.

\section{Ethics approval and consent to participate} Not applicable.

\section{Consent for publication \\ Not applicable.}

\section{Competing interests}

The authors declare no competing interests.

\section{Author details}

1 School of Pharmacy and Life Sciences, Robert Gordon University, Sir lan Wood Building, Garthdee Road, Aberdeen AB10 7GJ, Scotland. ${ }^{2}$ Graduate School, Robert Gordon University, The Ishbel Gordon Building, Garthdee Road, Aberdeen AB10 7QE, Scotland.

Received: 12 August 2020 Accepted: 9 December 2020

Published online: 06 January 2021

\section{References}

1. Bérdy J. Bioactive microbial metabolites. J Antibiot. 2005;58:1-26. https:// doi.org/10.1038/ja.2005.1.

2. Bérdy J. Thoughts and facts about antibiotics: where we are now and where we are heading. J Antibiot. 2012;65:385-95. https://doi. org/10.1038/ja.2012.27.

3. Lugtenberg B, Kamilova F. Plant-growth-promoting rhizobacteria. Annu Rev Microbiol. 2009;63:541-56. https://doi.org/10.1146/annurev.micro 62.081307 .162918

4. Rodríguez LA, Vivas J, Gallardo CS, Acosta F, Barbeyto L, Real F. Identification of Hafnia alvei with the MicroScan WalkAway system. J Clin Microbiol. 1999;37:4186-8. https://doi.org/10.1128/JCM.37.12.4186-4188.1999.

5. Legrand R, Lucas N, Dominique M, Azhar S, Deroissart C, Le Solliec MA, et al. Commensal Hafnia alvei strain reduces food intake and fat mass in obese mice-a new potential probiotic for appetite and body weight management. Int J Obes. 2020;44:1041-51. https://doi.org/10.1038/s4136 6-019-0515-9.

6. Günthard H, Pennekamp A. Clinical significance of Extraintestinal Hafnia alvei isolates from 61 patients and review of the literature. Clin Infect Dis. 1996;22:1040-5. https://doi.org/10.1093/clinids/22.6.1040. 
7. Tian B, Moran NA. Genome sequence of Hafnia alvei bta3_1, a bacterium with antimicrobial properties isolated from honey bee gut. Genome Announc. 2016;4:e00439-16. https://doi.org/10.1128/genomeA.00439-16.

8. Yin Z, Yuan C, Du Y, Yang P, Qian C, Wei Y, et al. Comparative genomic analysis of the Hafnia genus reveals an explicit evolutionary relationship between the species alvei and paralvei and provides insights into pathogenicity. BMC Genomics. 2019;20:768. https://doi.org/10.1186/s1286 4-019-6123-1.

9. Data File 1: Composition of ultra-minimal substrate growth medium. Figshare. 2020. https://doi.org/10.6084/m9.figshare.12781193.v1.

10. Bolger AM, Lohse M, Usadel B. Trimmomatic: a flexible trimmer for Illumina sequence data. Bioinformatics. 2014;30:2114-20. https://doi. org/10.1093/bioinformatics/btu170.

11. Andrews S. FastQC: a quality control tool for high throughput sequence data. http://www.bioinformatics.babraham.ac.uk/projects/fastqc/ (2010).

12. Ewels P, Magnusson M, Lundin S, Käller M. MultiQC: summarize analysis results for multiple tools and samples in a single report. Bioinformatics. 2016;32:3047-8. https://doi.org/10.1093/bioinformatics/btw354.

13. Data file 2: Quality distribution of Illumina reads. Figshare https://doi. org/10.6084/m9.figshare.12643961.v1 (2020)

14. Data file 3: Basic quality statistics of GridION sequencing data. Figshare https://doi.org/10.6084/m9.figshare.12781280.v1 (2020).

15. De Coster W, D'Hert S, Schultz DT, Cruts M, Van Broeckhoven C. NanoPack: visualizing and processing long-read sequencing data. Bioinformatics. 2018;34:2666-9. https://doi.org/10.1093/bioinformatics/bty149.

16. Wick RR, Judd LM, Gorrie CL, Holt KE. Unicycler: resolving bacterial genome assemblies from short and long sequencing reads. PLoS Comput Biol. 2017;13:1-22. https://doi.org/10.1371/journal.pcbi.1005595.

17. Gurevich A, Saveliev V, Vyahhi N, Tesler G. QUAST: quality assessment tool for genome assemblies. Bioinformatics. 2013;29:1072-5. https://doi. org/10.1093/bioinformatics/btt086.

18. Data file 4: Quast report. Figshare https://doi.org/10.6084/m9.figsh are.12781289.v1. (2020).

19. Simão FA, Waterhouse RM, loannidis P, Kriventseva EV, Zdobnov EM BUSCO: assessing genome assembly and annotation completeness with single-copy orthologs. Bioinformatics. 2015;31:3210-2. https://doi. org/10.1093/bioinformatics/btv351.

20. Data file 5: Short BUSCO summary. Figshare. 2020. https://doi. org/10.6084/m9.figshare.12643937.v1.

21. Jain C, Rodriguez-R LM, Phillippy AM, Konstantinidis KT, Aluru S. High throughput ANI analysis of 90K prokaryotic genomes reveals clear species boundaries. Nat Commun. 2018;9:5114. https://doi.org/10.1038/ s41467-018-07641-9.

22. Tatusova T, DiCuccio M, Badretdin A, Chetvernin V, Nawrocki EP, Zaslavsky $L$, et al. NCBI prokaryotic genome annotation pipeline. Nucleic Acids Res. 2016;44:6614-24. https://doi.org/10.1093/nar/gkw569.
23. Brettin T, Davis JJ, Disz T, Edwards RA, Gerdes S, Olsen GJ, et al. RASTtk: a modular and extensible implementation of the RAST algorithm for building custom annotation pipelines and annotating batches of genomes. Sci Rep. 2015;5:8365. https://doi.org/10.1038/srep08365.

24. Kanehisa M, Goto S. KEGG: kyoto encyclopedia of genes and genomes. Nucleic Acids Res. 2000;28:27-30. https://doi.org/10.1093/nar/28.1.27.

25. Huerta-Cepas J, Forslund K, Coelh LP, Szklarczyk D, Jensen LJ, von Mering $\mathrm{C}$, et al. Fast genome-wide functional annotation through orthology assignment by eggNOG-mapper. Mol Biol Evol. 2017;34:2115-22. https:// doi.org/10.1093/molbev/msx148.

26. Blin K, Shaw S, Steinke K, Villebro R, Ziemert N, Lee SY, et al. antiSMASH 50: updates to the secondary metabolite genome mining pipeline. Nucleic Acids Res. 2019;47:W81-7. https://doi.org/10.1093/nar/gkz310.

27. Stothard P, Grant JR, Van Domselaar G. Visualizing and comparing circular genomes using the CGView family of tools. Brief Bioinform. 2019;20:1576-82. https://doi.org/10.1093/bib/bbx081.

28. Data file 6: Circular representation of H. alvei A23BA genome. Figshare. https://doi.org/10.6084/m9.figshare.12643949.v1 (2020).

29. Data file 7: Predicted smBGCs of interest in H. alvei A23BA genome. Figshare. https://doi.org/10.6084/m9.figshare.12781274.v1 (2020).

30. Just-Baringo X, Albericio F, Álvarez M. Thiopeptide antibiotics: retrospective and recent advances. Mar Drugs. 2014;12:317-51. https://doi. org/10.3390/md12010317.

31. Robinson SL, Christenson JK, Wackett LP. Biosynthesis and chemical diversity of $\beta$-lactone natural products. Nat Prod Rep. 2019;36:458-75. https:// doi.org/10.1039/c8np00052b.

32. Saha M, Sarkar S, Sarkar B, Sharma BK, Bhattacharjee S, Tribedi P. Microbial siderophores and their potential applications: a review. Environ Sci Pollut Res Int. 2016;23:3984-99. https://doi.org/10.1007/s11356-015-4294-0.

33. Data file 8: Other gene clusters of interest in $\mathrm{H}$. alvei A23BA genome. Figshare. https://doi.org/10.6084/m9.figshare.12781181.v1 (2020).

34. Awolope, OK, Di Salvo A, O'Driscoll NH, Lamb AJ. Hafnia alvei strain A23BA chromosome, complete genome. GenBank. https://identifiers.org/ insdc:CP050150 (2020).

35. National Center for Biotechnology Information. Sequence Read Archive. https://identifiers.org/ncbi/insdc.sra:SRP251948 (2020).

36. National Center for Biotechnology Information. Assembly. https://ident ifiers.org/ncbi/insdc.gca:GCF_011617105.1 (2020).

\section{Publisher's Note}

Springer Nature remains neutral with regard to jurisdictional claims in published maps and institutional affiliations.
Ready to submit your research? Choose BMC and benefit from:

- fast, convenient online submission

- thorough peer review by experienced researchers in your field

- rapid publication on acceptance

- support for research data, including large and complex data types

- gold Open Access which fosters wider collaboration and increased citations

- maximum visibility for your research: over $100 \mathrm{M}$ website views per year

At BMC, research is always in progress.

Learn more biomedcentral.com/submissions 